\title{
CFD Simulation of Sodium Boiling in Heated Pipe using RPI Model
}

\author{
M. Naveen Raj, K. Velusamy \\ Thermal Hydraulics Section \\ Indira Gandhi Centre for Atomic Research, HBNI \\ Kalpakkam 603102, India \\ naveen@igcar.gov.in; kvelu@igcar.gov.in
}

\begin{abstract}
CFD prediction of sodium flow boiling in a 2-D asymmetric heated pipe is carried out using RPI wall heat flux partitioning model available in the commercial CFD code FLUENT. The two phase models are validated with published pressurized water and R12 flow boiling experimental data. The influence of radial mesh density on validation parameter is investigated. Void fraction, wall temperature and liquid temperature at different axial section are taken as the validation parameter for water experiment. Among the various parameters predicted, the void fraction of water at the outlet gave the largest deviation of 35\% with experimental data. But its value is in close comparison near the location of boiling initiation. Further the void fraction calculated based on heat balance compares well with the CFD results. Radial distributions of void fraction, vapour velocity, bubble diameter at the end of heated section are the parameters compared with R12 experimental data. Except bubble diameter, all other parameters gave reasonably good comparison. But when invoking breakup and coalescence model the predicted value of bubble diameter value approaches experimental data. Finally, sodium boiling simulations were carried out with the chosen models. It was found that, unlike water and R12, in the case of sodium, stable boiling was not achieved and boiling is highly localized to the end of heated section. Periodic growth and re-condensation of sodium vapour is found. Further, temporal oscillation in the heat transfer coefficient up to $8 \%$ was observed in the boiling zone. During sodium bubble expansion the evaporative heat flux dominates and once the bubble detaches, the quenching heat flux dominates. Unlike pressurised water evaporative and quenching heat flux have almost equal participation in boiling region.
\end{abstract}

Keywords: RPI model, CFD, sodium boiling, pulsating boiling

\section{Introduction}

In a fast breeder nuclear reactor, the heat generating fuel pins have a large heat flux $\sim 2 \mathrm{MW} / \mathrm{m}^{2}$ and each fuel bundle has 217 pins, where each pin is separated by a few millimetres by helically wound spacer wires. To remove such dense heat generated within the pin bundles, liquid sodium is used as an effective coolant due to its high thermal conductivity. During normal operating condition, sodium is maintained with large boiling margin $\left(<250^{\circ} \mathrm{C}\right)$. But during unwelcomed situations such as, blockage in the flow channel or reduction in the flow rate, sodium may reach boiling point. Consequence of such scenario should be well understood for optimal handling of such situations and to enhance thermal hydraulics design of future reactors. To investigate the onset and propagation of sodium boiling within pin bundles, various experiments have been carried out by different authors. Some of previous experimental investigations of sodium boiling are Kikuchi et al., [1], Khafizov et al. [2], Qiu et al. [3], Zicheng et al. [4] and Katsuro et al. [5]. But since sodium experiments are expensive and involve safety concerns, large volumes of numerical simulations were also reported on sodium boiling. The reported numerical simulations on sodium boiling are generally based on lumped approaches such as porous body [6] or subchannel analysis [7]. Recently, Vyskocil and Macek [8] performed 2D CFD simulation of R12 boiling in pipe flow using RPI model using FLUENT and NEPTUNE codes. Further, they compared the predicted results with the DEBORA experimental data [9]. Krepper et al. [10] simulated boiling in pressurized water using RPI model in CFX codes and validated it with experimental data reported by Bartolomej and Chanturiya [11]. They also discussed on the applicability of the boiling models to reactor pin bundle studies. Contrary to pressurized water and R12, sodium has large liquid to vapour density ratio and high thermal conductivity. In addition, from the sodium boiling experiment conducted by Katsuro et al. [5], they found that except under certain special circumstances, sodium generally tend to show pulsating boiling phenomenon with either single bubble expulsion or slug type expulsion. Though CFD based boiling simulations using RPI model were reported for water and R12, its applicability to sodium is not reported in published literatures. 
Further the influence of radial mesh density on the predicted two phase results has to be established for appropriate two phase and boiling modelling. These form the motivations for the present work.

\section{Mathematical Modelling}

In Eulerian multiphase model available in the commercial CFD code FLUENT, separate continuity and momentum equations are solved for each phase. But, energy equation is solved only for the liquid phase, where as the vapour phase is assumed to be at saturation temperature. The energy and momentum interfacial coupling terms are modelled using correlations listed in Table 1. Turbulence is modelled for each phase separately using Realizable k-e model with standard wall function. Here boiling is modelled based on RPI wall heat flux portioning model. The heat flux from the wall is resolved into three components such as the convective, quenching and evaporative heat fluxes. Again these components are calculated from flow and temperature fields along with other correlations for nucleation site density, departure frequency, and departure diameter shown in Table 1.

Second order upwind is used for discritization of momentum, energy, turbulent kinetic energy and dissipation in case of pressurized water and R12. But due to convergence issues first order upwind is used in the case of sodium. The residues were calculated based on local scaling. In R12 and sodium cases the residue fell below 1e-4, while for pressurised water cases it went below 1e-3 and stayed saturated.

Table 1: Models for Interfacial exchange terms used in present simulation.

\begin{tabular}{|l|l|}
\hline Physics & Model \\
\hline Drag force & Ishii model \\
\hline Lift force & Moraga model \\
\hline Virtual mass force & Explicitly derived \\
\hline Turbulent dispersion & Lopez de Bertodano Model \\
\hline Heat Exchange Coefficient & Ranz-Marshall Model \\
\hline Bubble departure diameter & Unal model \\
\hline Departure frequency & Cole model \\
\hline Nucleation side density & Lemmert-Chawla model \\
\hline Interfacial area concentration & Symmetric model \\
\hline Breakup and coalescence & Yao and Morel \\
\hline
\end{tabular}

\section{Validation}

The models used in the present analysis are compared with pressurized water and R12 experimental data reported in [11] and [9] respectively. For pressurized water validation, section averaged data are taken for comparison, whereas in R12 experiment radial distribution of various parameters at the end of heated section is taken as validation parameter. Further investigation was carried out to understand the influence of radial mesh density on various validation parameters.

\subsection{Validation with pressurized water experiment}

The dimensions and boundary conditions of the Bartolomej and Chanturiya [11] experimental setup are given in the Table 2. The axial mesh division is fixed at 521, while radial mesh is varied to assess the dependency of the results on the mesh size. Comparison of experimental data of area averaged void fraction, mass averaged liquid temperature, wall temperature with the simulated results for various radial mesh density is shown in Fig. 1. Further the void fraction 
value calculated from the quality determined by heat balance at any axial location is plotted in Fig. 1. Though the area averaged void fraction gave an over prediction of $35 \%$ with experimental data at the outlet, the comparison is reasonable near the location of boiling initiation. But the CFD value compares well with the calculated vapour fraction determined by heat balance. Liquid temperature compares very well with the experimental data and no significant difference was found between results predicted by different radial meshes. The wall temperature predicted compares well with the measured data with a maximum deviation of $5^{\circ} \mathrm{C}$. Even for large radial mesh variation, the maximum variation observed in void fraction is less than $28 \%$.

Table 2: Dimensions and Boundary condition of experimental setup and present analysis.

\begin{tabular}{|l|l|l|l|}
\hline Parameter & $\begin{array}{l}\text { Bartolomej and } \\
\text { Chanturiya [1] }\end{array}$ & DEBORA experiment [9] & Sodium simulation \\
\hline Fluid & Pressurized water & R12 & Sodium \\
\hline Length & Heated $=2 \mathrm{~m}$ & $\begin{array}{l}\text { Inlet adiabatic }=2 \mathrm{~m} \\
\text { Heated }=3.5 \mathrm{~m} \\
\text { Outlet adiabatic }=0.5 \mathrm{~m}\end{array}$ & $\begin{array}{l}\text { Heated }=1 \mathrm{~m} \\
\text { Outlet adiabatic }=0.3 \mathrm{~m}\end{array}$ \\
\hline Inner diameter & $15.4 \mathrm{~mm}$ & $19.2 \mathrm{~mm}$ & $40 \mathrm{~mm}$ \\
\hline Heat flux & $570000 \mathrm{~W} / \mathrm{m}^{2}$ & $58260 \mathrm{~W} / \mathrm{m}^{2}$ & $1650000 \mathrm{~W} / \mathrm{m} 2$ \\
\hline Operating pressure & $45 \mathrm{bar}$ & $30.06 \mathrm{bar}$ & $1 \mathrm{bar}$ \\
\hline Mass flux & $900 \mathrm{~kg} / \mathrm{m}^{2} / \mathrm{s}$ & $1006.8 \mathrm{~kg} / \mathrm{m}^{2} / \mathrm{s}$ & $312.3 \mathrm{~kg} / \mathrm{m} 2 / \mathrm{s}$ \\
\hline Inlet subcooling & $58.2 \mathrm{~K}$ & $41.17 \mathrm{~K}$ & $477 \mathrm{~K}$ \\
\hline
\end{tabular}

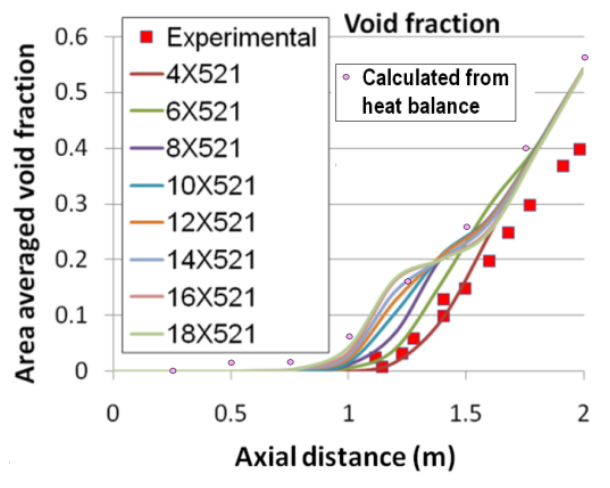

(a)

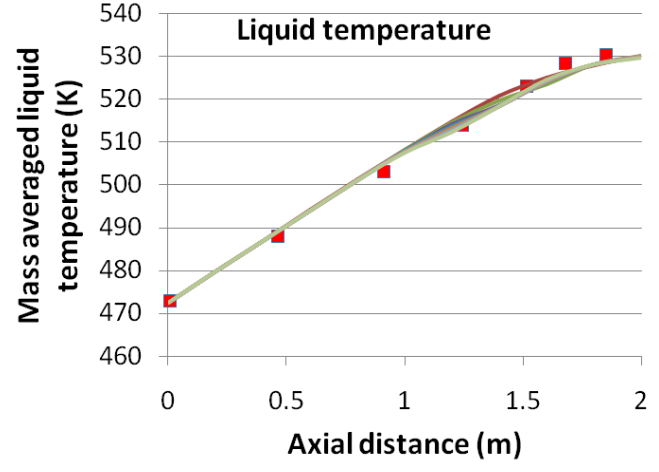

(b)

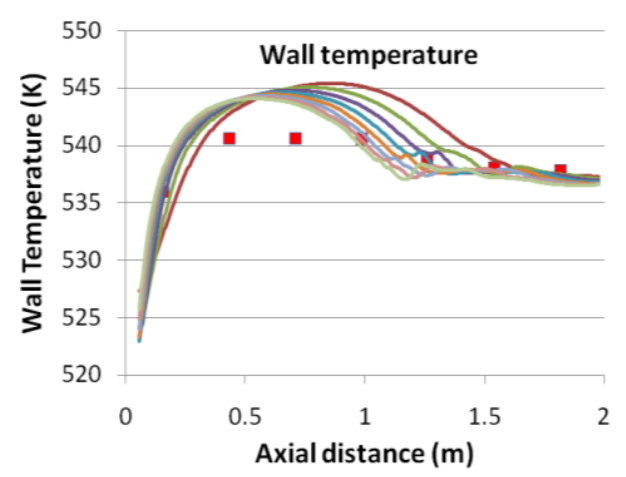

(c)

Fig. 1: Comparison of CFD predictions with pressurized water experimental data [11].

\subsection{Validation with $R 12$ experiment}

More crucial validation of the two phase models is done by comparing the radial gradients of various parameters obtained from Debora experiment [9]. The dimensions and boundary conditions of the Debora experimental setup is given in the Table 2. Initially the simulation is done without using breakup and coalescence model. Fixing the axial mesh at 1000 , radial mesh is varied. Comparison of experimental data of radial distribution void fraction, vapour velocity, bubble diameter with the simulated results for various radial mesh densities is shown in Fig. 2. Large radial meshes of 32 gave 
very good comparison with void fraction experimental data, but in general mesh above 10 gave $<25 \%$ under prediction. For all radial mesh used, vapour velocity compares well within $20 \%$. The predicted bubble diameter is more than twice the experimental data, and this is same for all the meshes. After formation, bubbles would have collapsed or broken up in the downstream. To include this phenomenon, interfacial area concentration is accounted using breakup and coalescence model based on Yao and Morel model and the results are presented in Fig. 3. It could be seen that when breakup and coalescence are considered, significant change was not observed in void fraction and vapour velocity, but bubble diameter came closer to experimental data.

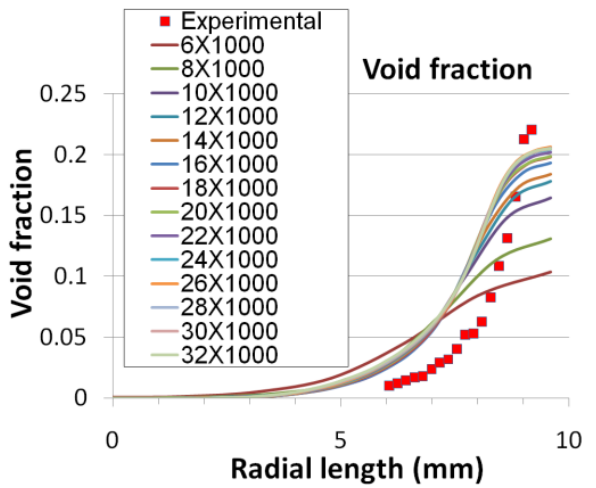

(a)

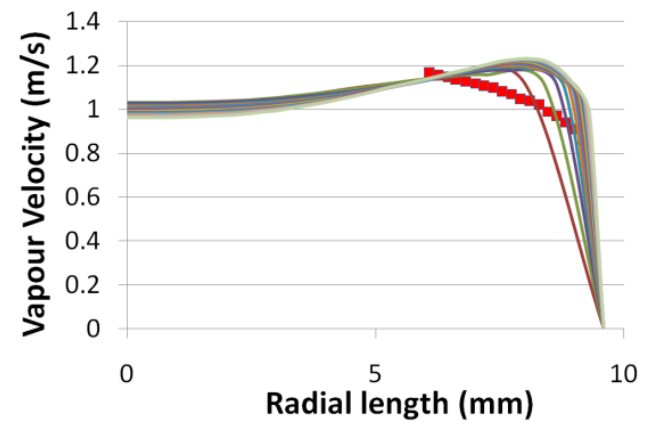

(b)

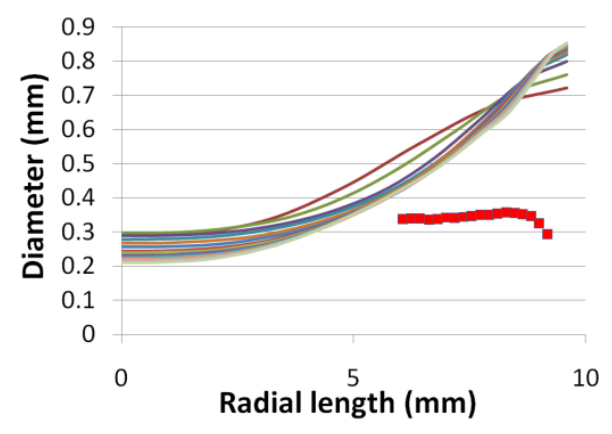

(c)

Fig. 2: Comparison of CFD predictions with R12 experimental data [9].

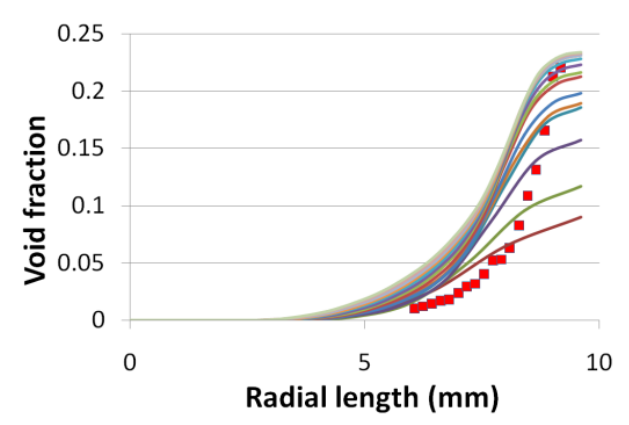

(a)

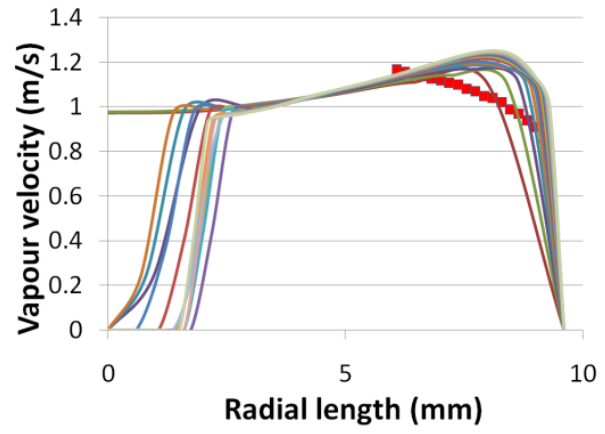

(b)

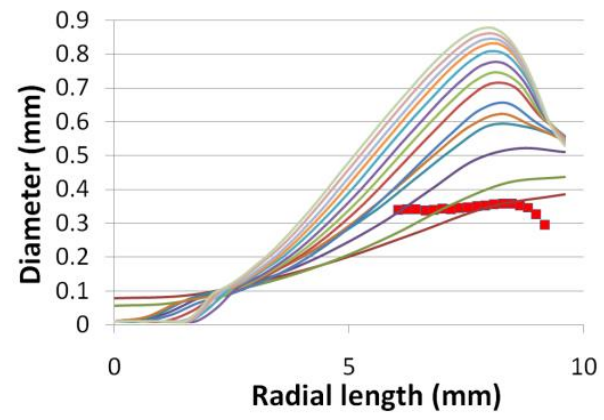

(c)

Fig. 3: Comparison of CFD predictions obtained with breakup and coalescence model, with R12 experimental data [9].

\section{Sodium boiling simulation}

After validating the various models involved in two phase heat transfer and fluid flow, sodium boiling in surface heated circular pipe is simulated. Though breakup and coalescence model gave closer comparison for bubble diameter, it is not used in the present simulation to avoid difficulty in convergence and uncertainty in application of the model to sodium. The dimensions and boundary condition taken for this analysis is shown in Table. 2. For the present simulation the number of mesh in axial and radial are taken as 650 and 10 respectively. The heat flux is applied only after the flow becomes completely steady within the domain after $\sim 60 \mathrm{~s}$. This is done since flow development along the length of the pipe requires larger time scale (i.e., $\sim 50 \mathrm{~s}$ ) compared to the boiling pulsation time scale (i.e. $\sim 0.1 \mathrm{~s}$ ). So zero in the plots corresponds to time after application of heat flux. The peak void fraction at the end of heated section and at $100 \mathrm{~mm}$ upstream of end of the heated section is shown in Fig. 4a. It was found that up to heat flux of 1.6 $\mathrm{MW} / \mathrm{m}^{2}$, the peak void fraction attained a stable value which is time invariant. But when the heat flux was raised to $1.65 \mathrm{MW} / \mathrm{m}^{2}$ the pulsation in void fraction was observed. This could be the critical flux when, bubble detachment occurs. The prediction of such pulsating boiling phenomenon is in compliance with the findings from sodium boiling experiments reported by Katsuro et al. [5]. The boiling simulation could be carried out only up to duration of $0.3 \mathrm{~s}$. 
The heat transfer coefficient at the end of heated section and at $100 \mathrm{~mm}$ upstream of it is shown in Fig. $4 \mathrm{~b}$. Oscillations up to $8 \%$ of its mean value is found in the heat transfer coefficient in the boiling region. Further, on an average, heat transfer coefficient in boiling region is $21 \%$ larger than the single phase heat transfer coefficient at $100 \mathrm{~mm}$ upstream of boiling region. Each cycle of heat transfer coefficient has a sharp trough in the middle separating two peaks. The first peak corresponds to the duration of expansion of bubble. The second peak corresponds to the duration after bubble detachment. The sharp tough in middle could be due to some numerical disturbance during the process of bubble detachment. Further it was found that the maximum oscillation in wall temperature in the boiling region is $22 \mathrm{~K}$. And on an average the wall temperature stays at $1162 \mathrm{~K}$, which corresponds to a wall super heat of $12 \mathrm{~K}$, since the boiling point of sodium is taken to be $1150 \mathrm{~K}$.

Contour plot of sodium void fraction at the instant when void fraction peaks is shown in Fig. 5. Steady state void fraction of pressurized water is also shown in Fig. 5. It is seen that sodium boiling occurs only at the end of the heated section. The boiling process in sodium is found to involve cyclic formation, growth and collapse of bubbles. But in the case of water the produced vapour bubbles continue to stay till the outlet. Immediate collapse of sodium vapour is largely due to high thermal conductivity of sodium, which quickly dissipates the heat within the bubble and allows it to condense.

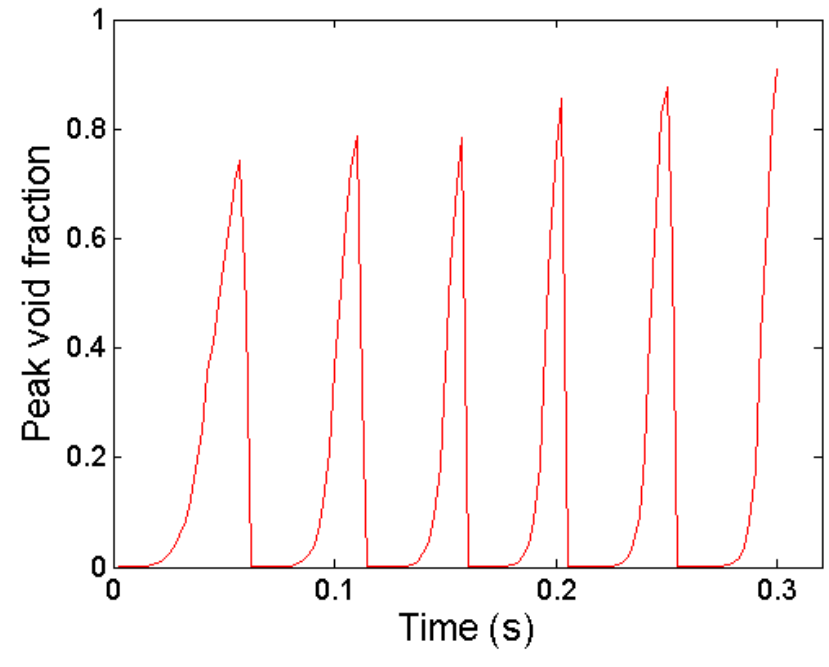

(a)

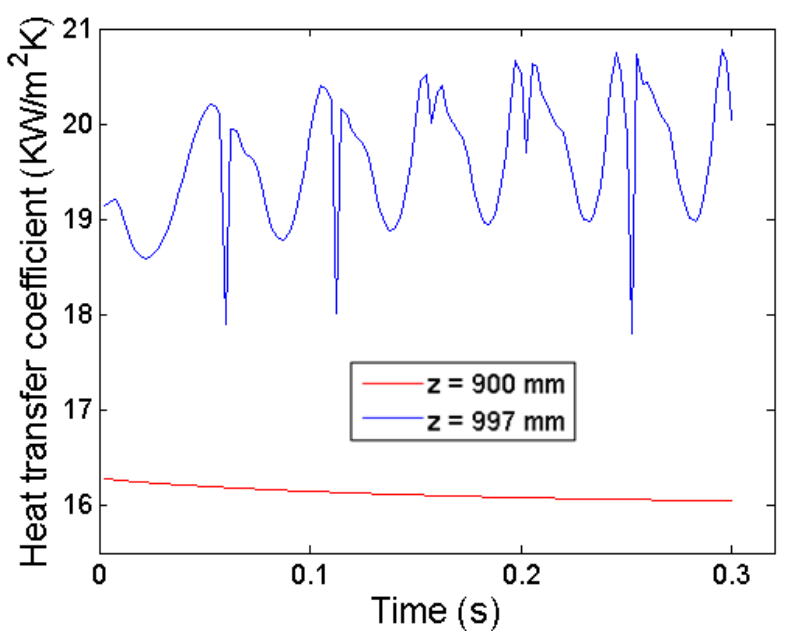

(b)

Fig. 4: (a) Peak void fraction and (b) Heat transfer coefficient at end of heated section ( $\mathrm{z}=997 \mathrm{~mm}$ ) and at $\mathrm{z}=900 \mathrm{~mm}$.

\subsection{Heat flux partitioning}

Different components of heat flux (i.e., evaporative, quenching, convective) for the case of pressurised water simulation and sodium are depicted in Fig. 6. In the case of water since there was no temporal variation, spatial distribution of heat flux components is show in Fig. 6a. On contrary, in case of sodium since the boiling is highly localized, the temporal variation of heat flux components only at the boiling region is shown in Fig. 6b. In the case of water, for the initial $200 \mathrm{~mm}$, the whole contribution of heat flux is from single phase convection, then slowly quenching component increases and reaches a maximum fraction of 0.5 and then drops. Beyond this region evaporative heat flux dominates and convective flux reduces to zero. Referring to Fig. $4 \mathrm{a}$ and $6 \mathrm{~b}$, in the case of sodium, the evaporative flux rises with rise in vapour fraction and becomes abruptly zero along with vapour fraction. The disturbance created by the detachment of the bubble causes sudden rise in quenching component beyond the drop in evaporative component. Then quenching component gradually reduces along with increase in the next phase of evaporation. Successive repetition of this cycle can be observed in Fig. 6b. For a very short period after bubble detachment, emergence of convective component could be observed, which otherwise stays at zero. 

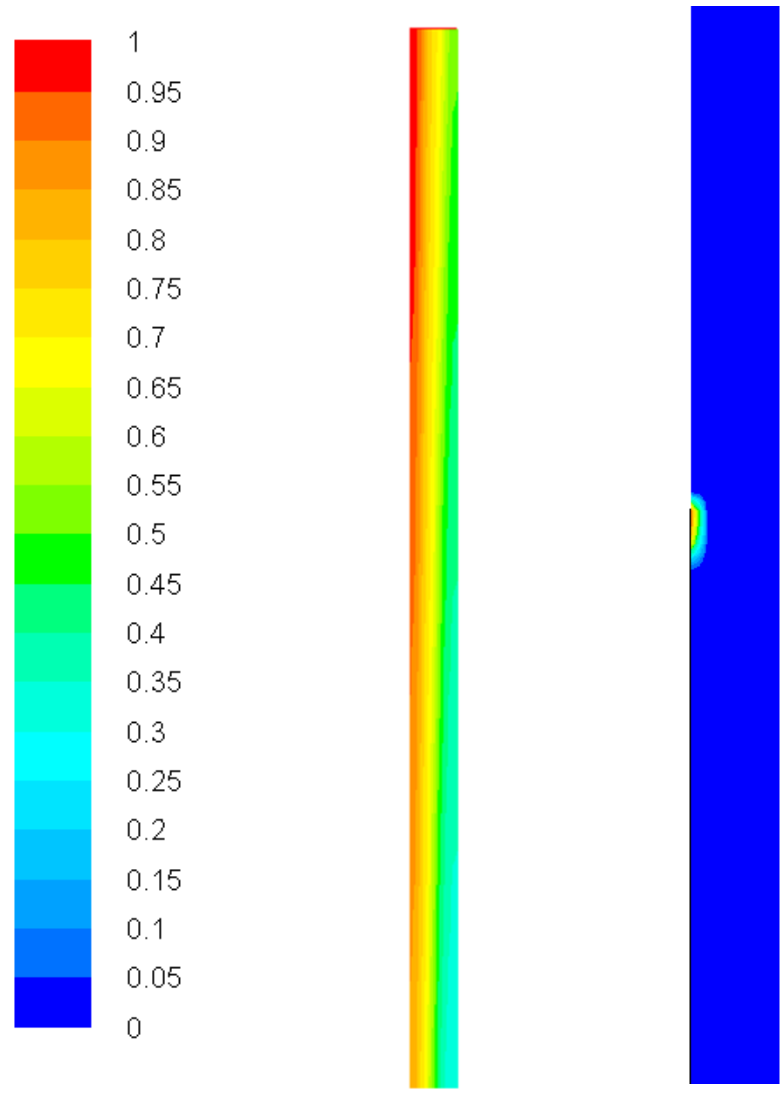

(a)

(b)

Fig. 5: Void fraction contour in (a) Water and (b) Sodium in the vicinity of end of heated section (marked in black line).

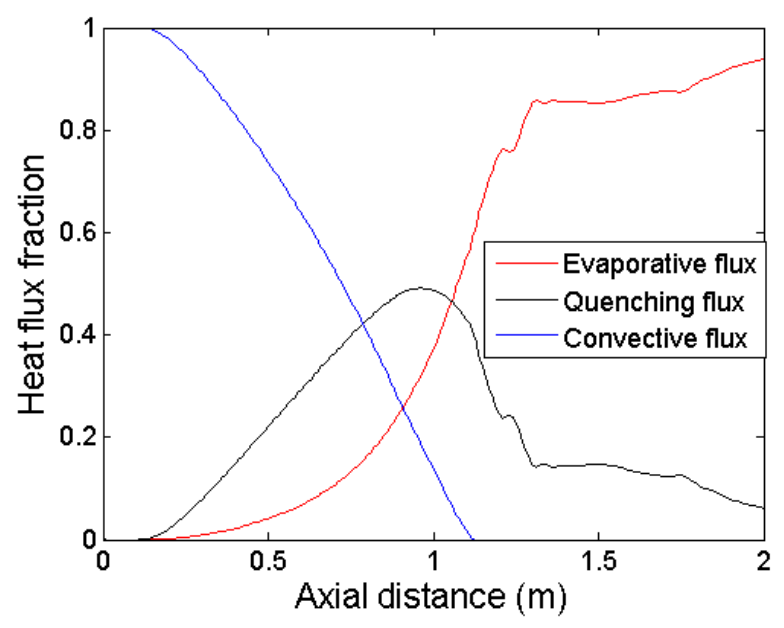

(a)

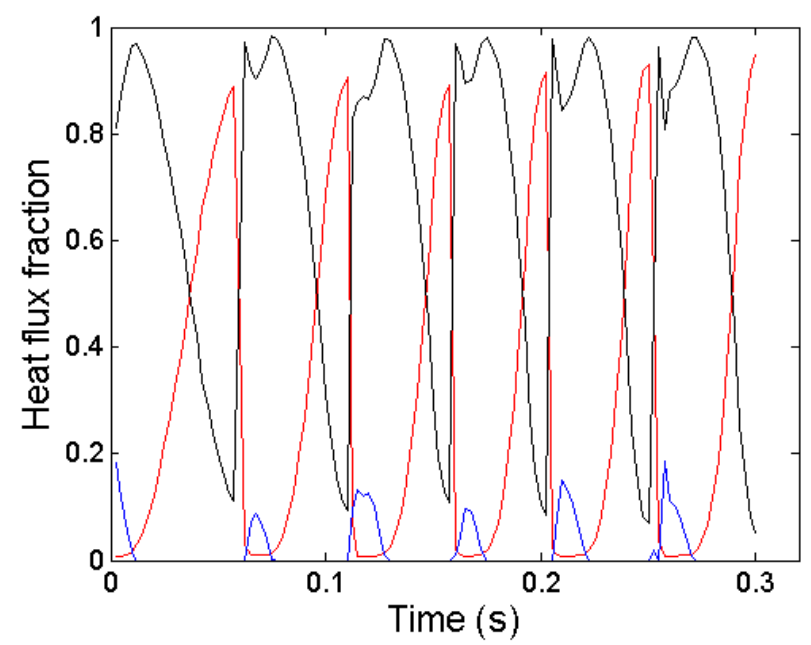

(b)

Fig. 6: Different components of heat flux (a) as a function of axial distance in pressurized water (b) at the end of heated section as a function of time in sodium. 


\section{Conclusion}

Towards understanding the boiling phenomena of sodium in pipe flow, CFD simulation is carried out using Eulerian multiphase model along with RPI wall heat flux portioning model. The various models handling the interfacial terms are validated with experimental data available for pressurized water and R12 refrigerant. In addition, sensitivity of the results to radial mesh size has also been investigated. In the case of water, among the various parameters predicted the void fraction at the outlet gave the largest deviation of $35 \%$ with experimental data. But its value is in close comparison near the location of boiling initiation. Further the void fraction calculated based on heat balance compares well with the CFD results. In the case of R12, bubble diameter exhibited a large deviation with experimental data. But when breakup and coalescence model is invoked the bubble diameter approached the experimental value. Sodium boiling simulation was carried out with the validated model. On contrary to pressurized water and R12, sodium showed pulsating boiling where periodic bubble formation, growth and collapse occurs. Further an $8 \%$ oscillation in heat transfer coefficient was observed in the vicinity of sodium boiling and average boiling heat transfer coefficient is $21 \%$ higher than the single phase heat transfer coefficient. The wall temperature in the vicinity of boiling is also found to oscillate by $22 \mathrm{~K}$ with an average wall superheat of $12 \mathrm{~K}$. In the vicinity of sodium boiling, both evaporative and quenching heat flux components have almost equal participation, with each component successively dominating alternate instances. The present analysis establishes the possibility of extending the RPI based boiling simulations to liquid metals (sodium) in pipe. Further investigation has to be carried out to improve the compliance of the model with liquid metal experimental data and to extend the simulation to boiling in reactor pin bundle.

\section{References}

[1] Y. Kikuchi, K. Haga, T. Takahashi, "Experimental study of steady state boiling of sodium flowing in a single pin annual channel," Journal of Nuclear Science and Technology, vol. 12, no. 2, pp. 83-91, 1975.

[2] R.R. Khafizov et al., "Experimental investigation of sodium boiling heat exchange in fuel subassembly mock-up for perspective fast reactor safety substantiation," Nuclear Energy and Technology, vol. 1, pp. 147-152, 2016.

[3] Z. C. Qiu et al., "Experimental research on the thermal hydraulic characteristics of sodium boiling in an annulus," Experimental Thermal and Fluid Science, vol. 60, pp. 263-274, 2015.

[4] Z. Qiu et al., "Experimental research on the incipient boiling wall superheat of sodium," Progress in Nuclear Energy, vol. 68, pp. 121-129, 2013.

[5] K. Takahashi, Y. Fujii, T. Suita, "Continous boiling phenomenon of sodium under forced convection by direct heating," Journal of Nuclear Science and Technology, vol. 9, no. 11, pp. 670-679, 1972.

[6] A. Chenu et al., "TRACE simulation of sodium boiling in pin bundle experiments under loss-of-flow conditions," Nuclear Engineering and Design, vol. 239, pp. 2417-2429, 2009.

[7] H. Ninokata, T. A. Okano, "SABENA: Subassembly Boiling Evolution Numerical Analysis," Nuclear Engineering and Design, vol. 120, pp. 349-367, 1990.

[8] V. Vyskocil, J. Macek, "Boiling Flow simulation in NEPTUNE CFD and FLUENT codes," CFD in Nuclear reactor safety - Proceeding of workshop on Experiments and CFD code application to nuclear reactor safety, vol. 44, no. 44.

[9] J. Garnier et al., "Local measurement on flow boiling of Refrigerant 12 in a vertical tube," Multiphase science and Technology, vol. 13, pp. 1-58, 2001.

[10] E. Krepper et al., "CFD modelling of subcooled boiling concept, validation and application to fuel assembly design," Nuclear Engineering and Design, vol. 237, pp. 716-731, 2007.

[11] G. G. Bartolomej, V. M. Chanturiya, "Experimental study of true void fraction when boiling subcooled water in vertical tube," Thermal Engineering, vol. 14, pp. 123-128, 1967. 